\title{
Glucose-6-Phosphate Dehydrogenase Deficiency and Adrenal Hemorrhage in a Filipino Neonate with Hyperbilirubinemia
}

\author{
Akira Ohishi, MD ${ }^{1}$ Daizo Ueno, MD ${ }^{1}$ Tsutomu Ogata, MD ${ }^{1}$ \\ ${ }^{1}$ Department of Pediatrics, Hamamatsu University School of \\ Medicine, Hamamatsu, Japan \\ Am J Perinatol Rep 2013;3:5-8. \\ Address for correspondence Akira Ohishi, MD, Department of \\ Pediatrics, Hamamatsu University School of Medicine, 1-20-1, \\ Handayama, Higashi-ku, Hamamatsu 431-3192, Japan \\ (e-mail: a-ohishi@hama-med.ac.jp).
}

\author{
Abstract \\ Keywords \\ - adrenal hemorrhage \\ - glucose-6-phosphate \\ dehydrogenase \\ deficiency \\ - hyperbilirubinemia \\ - neonate.
}

Neonatal hyperbilirubinemia is a common condition that usually ameliorates spontaneously. However, it can sometimes be retractile, especially when it is caused by some specific underlying diseases such as blood type incompatibility, sepsis, hepatitis, congenital biliary atresia, hemorrhage, and glucose-6-phosphate dehydrogenase (G6PD) deficiency. ${ }^{1,2}$ Thus, pediatricians must examine possible underlying factors in patients with severe, early onset, or prolonged hyperbilirubinemia. Here, we report a Filipino neonate with hyperbilirubinemia in whom co-occurrence of G6PD deficiency and adrenal hemorrhage was identified.

\section{Case Report}

This Filipino boy was born at 39 weeks of gestation at a local obstetric clinic. The pregnancy course was uneventful with no prenatal medications but the delivery was performed by a vacuum extraction due to an early rupture of membranes and prolonged labor. His birth length was $51.5 \mathrm{~cm}$, and his birth weight $4.19 \mathrm{~kg}$. At birth, he exhibited generalized cyanosis and received resuscitation with oxygen. His Apgar score was 6 at 1 minute and 7 at 5 minutes. A cord blood examination was not done in this clinic. Oral feeding with infant formula was started on day 0, despite tachypnea. At 45 hours

received

December 3, 2011

accepted after revision

June 20, 2012

published online

November 16, 2012

after birth, he was treated with single phototherapy because of early onset hyperbilirubinemia (serum total bilirubin $>17 \mathrm{mg} / \mathrm{dL}$ ).

At 54 hours after birth, he was referred to Hamamatsu University Hospital because of persistent tachypnea and jaundice. On admission, his respiratory rate was $70 / \mathrm{min}$, his skin appeared jaundiced, he was awake and alert, and there were no hepatosplenomegaly. Laboratory studies done at hospital inspection department showed increased serum total bilirubin $(19.8 \mathrm{mg} / \mathrm{dL}$ ) and unconjugated bilirubin (19.3 $\mathrm{mg} / \mathrm{dL}$ ), as well as elevated liver enzymes (aspartate amino transferase $164 \mathrm{IU} / \mathrm{L}$; alanine transaminase $70 \mathrm{IU} / \mathrm{L}$; lactate dehydrogenase $2906 \mathrm{IU} / \mathrm{L}$; creatine kinase $3267 \mathrm{IU} / \mathrm{L}$; and creatine $1.17 \mathrm{~g} / \mathrm{dL}$ ), which were consistent with stressful delivery and possible perinatal hypoxic-ischemic insult. Elevated liver enzymes and creatine kinase become diminished on next day. Hematologic studies showed that blood hemoglobin level was $18.5 \mathrm{~g} / \mathrm{dL}$, reticulocytes was 32 per mill, there were no crushed or immature blood cells and poikilocyte in peripheral smear, and his blood type group was $\mathrm{A} \mathrm{Rh}(+)$. Blood coagulation tests showed no abnormal level suggesting disseminated intravascular clotting.

He was immediately treated with oxygen, double phototherapy, and antibiotics. We used Murata et al's guidelines for

Copyright (c 2013 by Thieme Medical Publishers, Inc., 333 Seventh Avenue, New York, NY 10001, USA. Tel: +1(212) 584-4662.
DOI http://dx.doi.org/ 10.1055/s-0032-1329125. ISSN 2157-6998. 


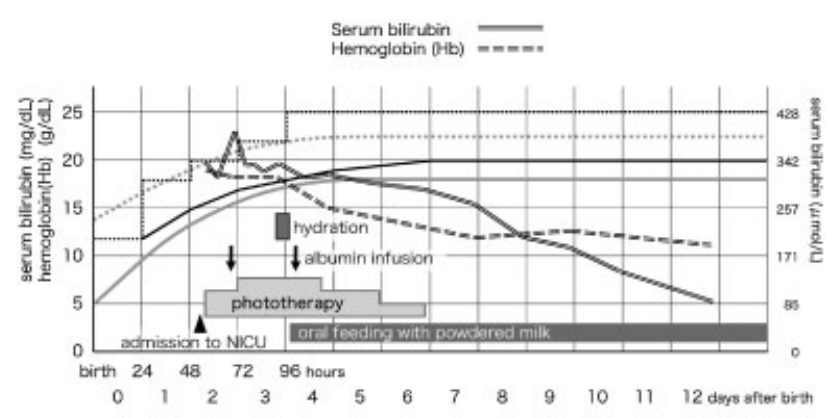

Limit line of exchange transfusion by Nakemura's guideline for infents over $2500 \mathrm{~g} . . . . . . . . . . .$. Limit line of phototherapy by Murata's guideline for infants over $2500 \mathrm{~g}$

Limit line of exchange transfusion by AAP for infants at medium risk Limit line of phototherapy by AAP for infants at medium ris

Fig. 1 Clinical course of this patient. We used Murata et al's guidelines for phototherapy ${ }^{3}$ and Nakamura's guidelines for exchange transfusion ${ }^{4}$ in conjunction. The patient was an infant with medium risk in the AAP guidelines ${ }^{1}$ because of possible perinatal hypoxic-ischemic insult. Phototherapy was done with PIT-250ICR (Atom Medical), neo Blue LED Phototherapy (Natus Medical Incorporated), and Bili-bracket plus high output (Ohmeda Medical, Laurel, MD, USA). NICU, neonatal intensive care unit; AAP, American Academy of Pediatrics.

phototherapy $^{3}$ and Nakamura's guidelines for exchange transfusion ${ }^{4}$ in conjunction with American Academy of Pediatrics guidelines. ${ }^{1}$ Maximum C-reactive protein $(1.28 \mathrm{mg} / \mathrm{dL})$, maximum white blood cell count $(13,000 / \mu \mathrm{L})$, and negative blood culture confirmed that there was no sepsis. Consequently, there was no air leak or permeability alteration in chest X-ray, and his tachypnea was mitigated within a few days, so delayed cardiopulmonary transition was diagnosed. However, while serum total bilirubin was transiently reduced to $18.4 \mathrm{mg} / \mathrm{dL}$ at 61 hours after birth, it reelevated to $23 \mathrm{mg} / \mathrm{dL}$ at 70 hours, and triple phototherapy and fluid infusion with
7.5\% dextrose solution to dilute serum bilirubin were started at 3 days of age. Although serum albumin was $3.3 \mathrm{mg} / \mathrm{dL}$ at that time, albumin infusion was used together to reduce unbound bilirubin (-Fig. 1). Oral feeding with infant formula was restarted. At that time, hemoglobin level was found to be reduced to $13.5 \mathrm{~g} / \mathrm{dL}$. With these therapies, serum total bilirubin was gradually reduced, and phototherapy was discontinued at 7 days of age in a stepwise fashion to avoid reelevation of serum bilirubin. Subsequent physical examinations showed no symptoms of bilirubin encephalopathy such as lethargic or hypotonic and suck poorly. Head magnetic resonance imaging (MRI) took before discharge our neonatal intensive care unit (NICU) revealed no unusual intensity of nucleus basalis and encephalomalacia that would suggest kernicterus or hypoxic-ischemic encephalopathy.

As a routine study for neonates admitted to our NICU, ultrasonographic survey was performed at the time of admission to investigate congenital heart disease, intraventricular hemorrhage, hydronephrotic kidney, and other anomalies. Consequently, a low echoic cystic mass of approximately $5 \times 3 \times 3 \mathrm{~cm}$ in size was identified at a ventral and temporal position of right kidney (-Fig. 2a). Subsequent follow-up studies with ultrasonography, computed tomography, and MRI delineated an inhomogeneous mass within right adrenal gland (-Figs. 2b, 2c). The tumor markers levels were within the normal range (serum neuron-specific enolase $21 \mathrm{ng} / \mathrm{mL}$, urine vanillylmandelic acid $0.8 \mathrm{mg} / \mathrm{L}$, and urine homovanillic acid $1.7 \mathrm{mg} / \mathrm{L}$ ), as were adrenal hormone levels (serum cortisol $8.3 \mu \mathrm{g} / \mathrm{dL}$ and $17 \alpha$-hydroxyprogesterone $3.4 \mathrm{ng} / \mathrm{dL}$ ). The mass steadily reduced in size and vanished on day 51. Thus, the mass was diagnosed as an adrenal hematoma. There was no other internal breading such as intracranial hemorrhage by ultrasonographic survey. There
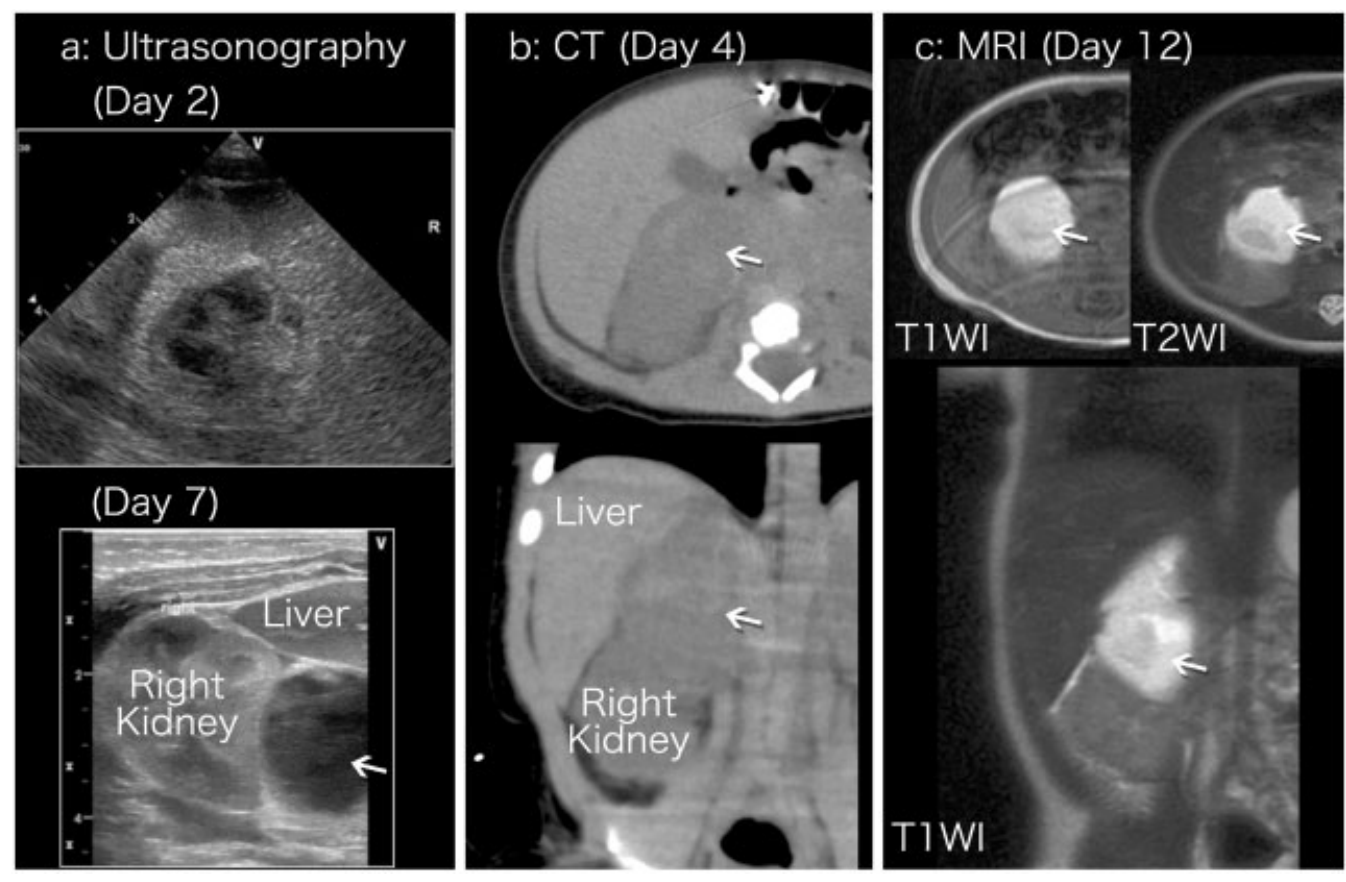

Fig. 2 Adrenal hemorrhage of this patient is indicated by arrows. An inhomogeneous mass is seen to the right of the adrenal gland. CT, computed tomography; MRI. magnetic resonance imaging. 
was no hypotension or hypoglycemia that suggests adrenal failure.

We examined serum G6PD activity and the G6PD gene on the $\mathrm{X}$ chromosome, because: (1) this patient was of Filipino origin with a high prevalence of G6PD deficiency, ${ }^{5}$ which is an important cause of hyperbilirubinemia, ${ }^{1,6,7}$ and (2) his one male sibling had hyperbilirubinemia and received phototherapy. The Formazan method ${ }^{8}$ done when the patient was 4 months of age revealed that his serum G6PD activity was $18 \%$ as compared with normal adult levels. Direct sequencing showed homozygosity for c.871G $>$ A (p.V291M) on exon 9 (the common Viangchan-type mutation); this mutation was associated with c.1311C $>$ T on exon 11 and IVS11 $+93 \mathrm{~T}>$ C, as reported previously. ${ }^{9}$ Similar studies were also performed for the mother, the fourth brother, and the fifth brother, indicating that the mother was heterozygous for the mutation and the fifth brother also had G6PD deficiency.

\section{Discussion}

We identified G6PD deficiency and adrenal hemorrhage in a Filipino neonate with hyperbilirubinemia. G6PD deficiency is a common congenital enzyme defect that is highly prevalent in the Filipino population, 5,6,10 and neonatal adrenal hemorrhage is a rare condition caused by several factors such as traumatic delivery, shock, and hypoxia. ${ }^{6,11}$ Because G6PD deficiency leads to red cell membrane breakage with various types of oxidation stress, this condition can cause hemolytic anemia and hyperbilirubinemia at 1 to 4 days of age in neonates with asphyxia. ${ }^{6,12,13}$ Furthermore, because adrenal hemorrhage as well as cephalic and other hemorrhages result in gradual hemolysis and resultant production of bilirubin, this condition can cause prolonged hyperbilirubinemia in neonates with asphyxia. ${ }^{2,14,15}$ Thus, the co-occurrence of G6PD deficiency and adrenal hemorrhage would explain the early onset and prolonged hyperbilirubinemia of this Filipino neonate. In addition, the time gap for the bilirubin production caused by G6PD deficiency and adrenal hemorrhage may explain why his hyperbilirubinemia was ameliorated by phototherapy without blood exchange transfusion.

In summary, we identified G6PD deficiency and adrenal hemorrhage in a Filipino neonate with hyperbilirubinemia. Notably, asphyxia appears to be relevant to the clinical manifestation of G6PD deficiency and the development of adrenal hemorrhage. Our results represent the importance of recognition of these risk factors and examining possible underlying clinical conditions for the development of severe, early onset, or prolonged hyperbilirubinemia. ${ }^{4}$ Although G6PD deficiency patients are distributed unevenly, ${ }^{8}$ in a globalized society, we should provide medical care without sticking to former ways of differential diagnosis and incidence of diseases. $^{12}$

\section{Acknowledgment}

We received no financial support for this case. We received support from Prof. Matsuoka Hiroyuki, Department of Medical Zoology at Jichi Medical University, Shimotsuke, Japan; and Prof. Fumihiko Kawamoto, Department of Infectious Disease Control at Oita University, Oita, Japan, for diagnosis of G6PD deficiency.

\section{References}

1 American Academy of Pediatrics, Subcommittee on Hyperbilirubinemia. Management of hyperbilirubinemia in the newborn infant 35 or more weeks of gestation [published correction appears in Pediatrics 2004;114:1138]. Pediatrics 2004;114: 297-316

2 Katar S, Oztürkmen-Akay H, Devecioğlu C, Taşkesen M. A rare cause of hyperbilirubinemia in a newborn: bilateral adrenal hematoma. Turk J Pediatr 2008;50:485-487

3 Murata B, Imura S, Kiuchi M, Takahashi S, Nemoto H. Phototherapy for neonatal hyperbilirubinemia: clinical problems in its use. Shoni geka, naika [Jap J Pediatr Surg and Med] 1973;5:301-311

4 Nakamura $H$. Management of hyperbilirubinemia. In: Kobe University Department of Pediatrics, eds. Management of Premature Infants and Newborns. Tokyo: Nihon shoni-iji shuppan-sha; 2000:225-240

5 Nkhoma ET, Poole C, Vannappagari V, Hall SA, Beutler E. The global prevalence of glucose-6-phosphate dehydrogenase deficiency: a systematic review and meta-analysis. Blood Cells Mol Dis 2009;42:267-278

6 Cappellini MD, Fiorelli G. Glucose-6-phosphate dehydrogenase deficiency. Lancet 2008;371:64-74

7 Frank JE. Diagnosis and management of G6PD deficiency. Am Fam Physician 2005;72:1277-1282

8 Tantular IS, Iwai K, Lin K, et al. Field trials of a rapid test for G6PD deficiency in combination with a rapid diagnosis of malaria. Trop Med Int Health 1999;4:245-250

9 Matsuoka H, Nguon C, Kanbe T, et al. Glucose-6-phosphate dehydrogenase (G6PD) mutations in Cambodia: G6PD Viangchan $(871 \mathrm{G}>\mathrm{A})$ is the most common variant in the Cambodian population. J Hum Genet 2005;50:468-472

10 Silao CL, Shirakawa T, Nishiyama K, Padilla C, Matsuo M. Molecular basis of glucose-6-phosphate dehydrogenase deficiency among Filipinos. Pediatr Int 1999;41:138-141

11 Qureshi UA, Ahmad N, Rasool A, Choh S. Neonatal adrenal hemorrhage presenting as late onset neonatal jaundice. J Indian Assoc Pediatr Surg 2009;14:221-223

12 Dhillon AS, Darbyshire PJ, Williams MD, Bissenden JG. Massive acute haemolysis in neonates with glucose-6-phosphate dehydrogenase deficiency. Arch Dis Child Fetal Neonatal Ed 2003;88: F534-F536

13 Kaplan M, Hammerman C. Glucose-6-phosphate dehydrogenase deficiency: a potential source of severe neonatal hyperbilirubinaemia and kernicterus. Semin Neonatol 2002;7:121-128

14 Gunlemez A, Karadag A, Degirmencioglu H, Uras N, Turkay S. Management of severe hyperbilirubinemia in the newborn: adrenal hematoma revisited. J Perinatol 2005;25:803-804

15 Velaphi SC, Perlman JM. Neonatal adrenal hemorrhage: clinical and abdominal sonographic findings. Clin Pediatr (Phila) 2001;40: $545-548$ 
